\title{
Avispas de la vegetación arbórea en el Parque Nacional Natural Gorgona, Pacífico de Colombia
}

\author{
Lorena Lopez $^{1 *}$, Stephany Valdés-Rodríguez ${ }^{1} \&$ Patricia Chacón de Ulloa ${ }^{1}$ \\ 1. Sección de Entomología, Facultad de Ciencias Naturales y Exactas, Departamento de Biología, Universidad del Valle, \\ Carrera100 Calle13 Cali, Colombia; lorelopezq.257@gmail.com, tefa.valdes@gmail.com, \\ patricia.chacon@correounivalle.edu.co
}

Recibido 18-X-2013. Corregido 20-XI-2013. Aceptado 19-XII-2013.

\begin{abstract}
Wasps from the arboreal vegetation in Gorgona National Natural Park, Colombian Pacific. Studies of Colombian Hymenoptera are incipient, fragmented and there is no comprehensive study about its size and composition. We know that the guild of hymenopteran parasitoids is the richest in species and that they are common and abundant in all terrestrial ecosystems, acting as regulators of populations of other insects. Studies in Gorgona National Natural Park have mostly focused on the natural history and identification of Formicidae and Apidae. This work was directed toward the identification of other hymenopteran species associated with its tropical rain forest. In November 2007, 16 trees were sampled by fogging with a biodegradable pyrethroid insecticide applied from $1 \mathrm{~m}$ above the ground to the canopy. We found 641 individuals ( $45 \%$ identified to subfamily and $28 \%$ to genera) from seven superfamilies, 20 families and 118 morphospecies. Chalcidoidea was the most representative superfamily, Eulophidae the most diverse family and Braconidae the numerically dominant family. The sampling efficiency was $71 \%$ and 166 species are estimated to exist in the island. Approximately $71 \%$ of the estimated species were captured during this study. We report 12 genera new for the island. Rev. Biol. Trop. 62 (Suppl. 1): 307-315. Epub 2014 February 01.
\end{abstract}

Key words: Chalcidoidea, waps, insular ecosystem, fogging technique, tropical rainforest.

Los himenópteros son hiperdiversos tanto en especies como en funciones y adaptaciones ecológicas (Lasalle \& Gauld, 1993). El gremio de los himenópteros parasitoides es el más rico en especies, son comunes y abundantes en todos los ecosistemas terrestres y actúan como reguladores de las poblaciones de otros insectos, siendo ésta una función biológica de gran importancia para el ecosistema (Lasalle, 1993). Los trabajos sobre este gremio se han enfocado en reconocer y utilizar especies para planes de manejo de plagas en áreas de producción agrícola (Madrigal-Cardeño, 2001; Ugarte-Lavados \& Dassori, 2009), estudios de movilidad en paisajes fragmentados y sotobosque (López, Armbrecht, Montoya-Lerma \& Molina, 2013), mientras que trabajos de identificación y caracterización de nuevas especies o aquellas pertenecientes a otro tipo de hábitats (áreas boscosas o bosques prístinos) han sido menos frecuentes (Gibson, Huber \& Woolley, 1997; Madrigal-Cardeño, 2001).

Conocer la estructura vegetal del área de interés es de gran importancia ya que diferentes especies de himenópteros pueden estar asociadas a diferentes estratos vegetales. El bosque húmedo tropical del Parque Nacional Natural Gorgona (PNN Gorgona), localizado en la región insular del océano Pacífico colombiano, se caracteriza por presentar en el estrato arbóreo especies dominantes como: Vismia baccifera, Cecropia garciae, Cespedesia macrophylla, Eschweilera pittieri, Perebea xanthochyma, Ardisia opaca, Myrcia fallax, Ryania speciosa, Zanthoxylum grandiflorum y Vochysia ferruginea, Cespedesia macrophylla y Symphonia globulifera (Rangel, 1990). En el estrato herbáceo se reporta Tara sp., Triolena 
spicata, Tetrorchidium gorgonae y Wedelia triloba (Rangel, 1990). Asimismo se reconoce la modificación de la estructura vegetal cerca de las playas, en el costado oriental y suroriental de la Isla, resultado del efecto de las actividades humanas durante el funcionamiento de la prisión (1959-1984) (Chamorro, 1990), encontrándose vestigios de plantaciones de cocales (Cocos nucifera) y algunos árboles frutales como: guayabos (Psidium guajava), limoncillo (Citrus medica), aguacate (Persea americana), mango (Manguifera indica), árbol del pan (Artocarpus altilis), entre otros (Rangel, 1990; Vásquez, Cortés-Ceballos, Calero-Rosales, Soto \& Torres, 2012).

Desde hace más de 20 años, se ha considerado el área del dosel como un microhábitat que contiene una gran diversidad de especies (Erwin \& Scott, 1980; Wilson, 1988; Gaston, 1991; Stork, 1993; Kitching, Mitchell, Morse \& Thebaud, 1997; Stork \& Hammond, 1997; Lucky, Erwin \& Witman, 2002; Basset, Hammond, Barrios, Holloway \& Miller, 2003), siendo uno de los métodos más efectivos y utilizados para el estudio de ésta diversidad la técnica de termonebulización o "fogging" aplicada con un insecticida no residual, que permite realizar colectas de forma rápida y sistemática (Erwin, 1982; Stork \& Hammond, 1997). Aunque en el PNN Gorgona se han realizado esfuerzos de investigación entomológicos (e.g. Andrade et al., 1990), en su mayoría han sido realizados con métodos tradicionales de muestreo y enfocados en la historia natural e identificación de especies de Formicidae y Apidae (Ulloa-Chacón, 1990; Baena, 1993; Ramírez, Dressler \& Ospina, 2002; Valdés-Rodríguez, Hurtado \& Chacón, 2010; González-Córdoba, González \& Montoya-Lerma, 2011), y algunos estudios faunísticos con especies de Braconidae y Ichneumonidae que reportan baja diversidad de especies (Arias-Penna, 2007; Fernández \& Sharkey, 2006; Pimienta, Montealegre, Chacón de Ulloa, González \& Mejía, 2008).

Recientemente, Pimienta et al. (2008) realizaron la revisión preliminar de los artrópodos arbóreos del PNN Gorgona, convirtiéndose en una de las pocas fuentes de información que contiene registros de himenópteros parasitoides de dosel en Colombia. Como respuesta al bajo número de registros de especies para esta localidad, en el presente trabajo se describe la fauna de himenópteros parasitoides del PNN Gorgona y se amplía el inventario taxonómico de ésta área protegida, aportando información técnica que incrementa el conocimiento sobre la biodiversidad de los ecosistemas insulares del Pacífico tropical.

\section{MATERIALES Y MÉTODOS}

Área de estudio: La isla Gorgona está situada dentro de la franja tropical del pacífico americano, al suroeste de Colombia, en el Departamento del Cauca (2॰56'34'-2 ${ }^{\circ} 58^{\prime} 44^{\prime}$ ' N y $78^{\circ} 11^{\prime} 08^{\prime \prime}-78^{\circ} 12^{\prime} 51^{\prime \prime}$ W) (Giraldo, 2012) y hace parte del sistema de Parques Nacionales Naturales de Colombia. Tiene una extensión territorial de $13.82 \mathrm{~km}^{2}$ y se conecta con la pequeña Isla de Gorgonilla $\left(0.49 \mathrm{~km}^{2}\right)$. Su máxima altura es $338 \mathrm{msnm}$ (Cerro Trinidad), tiene un promedio de precipitación anual de $6694 \mathrm{~mm}$, humedad relativa de $90 \%$, temperatura promedio de $27^{\circ} \mathrm{C}$ (Chamorro, 1990; Rangel, 1990; Cavelier \& Yockteng, 1998) y pertenece a la zona de vida de bosque muy húmedo tropical (Bmh-T) (Espinal \& Montenegro, 1977). La vegetación predominante es el bosque secundario con árboles de hasta $35 \mathrm{~m}$ de alto con familias de plantas como Bombacaceae, Mimosaceae, Cesalpiniaceae, Fabaceae, Polygonaceae, Euphorbiaceae, Rubiaceae y Monimiaceae, además se distinguen zonas de cultivos con comunidades pioneras e intermedias (Aguirre \& Rangel., 1990).

Método de muestreo: Se seleccionaron al azar 16 árboles del sector oriental de la isla, en el sitio denominado "sendero del tsunami" y detrás de la antigua prisión. Del 15 al 17 de noviembre de 2007 en éstos se instalaron sabanas colectoras de nylon $(2 \times 2 \mathrm{~m})$, y afuera a nivel del suelo o a diferentes alturas $(1 \mathrm{~m}, 5 \mathrm{~m}$, $10 \mathrm{~m}$ y más de $15 \mathrm{~m}$ ). En el centro de cada sábana se adaptó un recipiente con etanol al $80 \%$ en el cual se guardaron todos los artrópodos 
recolectados (Pimienta et al., 2008). Todos los árboles fueron muestreados entre las 00:00 y las 2:00h, utilizando un insecticida piretroide biodegradable (Permost VPM $®$ ) aplicado mediante la técnica de termonebulización, adaptada de Pimienta (2005).

Determinación de las muestras: Los especímenes del orden Hymenoptera exceptuando las familias Chalcididae y Formicidae, se identificaron hasta el nivel taxonómico más bajo posible utilizando las claves de Gibson et al. (1997), Fernández (2001) y Fernández \& Sharkey (2006). La colección de referencia se depositó en el Museo de Entomología de la Universidad del Valle (MUSENUV) (Cali-Colombia), códigos 23681-24020, 24034-24042.

Análisis de datos: Se realizó una análisis descriptivo y se determinó la eficiencia del muestreo empleando un promedio de tres estimadores no paramétricos basados en datos de presencia-ausencia: ICE, Chao2 y Jacknife1 (EstimateS v. 8.2, Colwell, 2012).

\section{RESULTADOS}

El total de himenópteros parasitoides recolectados en los 16 árboles del PNN Gorgona, comprendió siete superfamilias, 20 familias, 118 morfoespecies y 641 individuos. El 54\% de las morfoespecies se identificó hasta subfamilia y el $28 \%$ hasta género, siendo Chalcidoidea la superfamilia más representativa (diez familias y 63 morfoespecies) (Fig. 1). De la familia Braconidae se obtuvo la mayor abundancia (137 individuos pertenecientes a 16 morfoespecies), seguida por Platygastridae (135 individuos pertenecientes a 15 morfoespecies) y por Eulophidae (72 individuos) la cual registró el mayor número de morfoespecies (21) (Fig. 1).

Se lograron determinar 23 géneros de 11 familias (Cuadro 1). Sobresalen las familias Eurytomidae y Torymidae con cinco géneros cada una. De Mymaridae y Eucharitidae se identificaron tres géneros mientras que Agaonidae estuvo representada por dos géneros $\mathrm{y}$ Encyrtidae presentó un solo género con tres morfoespecies. De las siete morfoespecies de Pteromalidae solo se determinó una del género Dipachystigma. También para las familias Ceraphronidae, Dryinidae, Cynipidae y Figitidae se halló un género (Cuadro 1). De las otras dos familias de Chrysidoidea, se hallaron tres morfoespecies de Bethylidae (Pristocerinae, $\mathrm{n}=24$ ) y tres más de Crabronidae, representada por dos especies de la tribu Miscophini y una de Psenini (Cuadro 1).

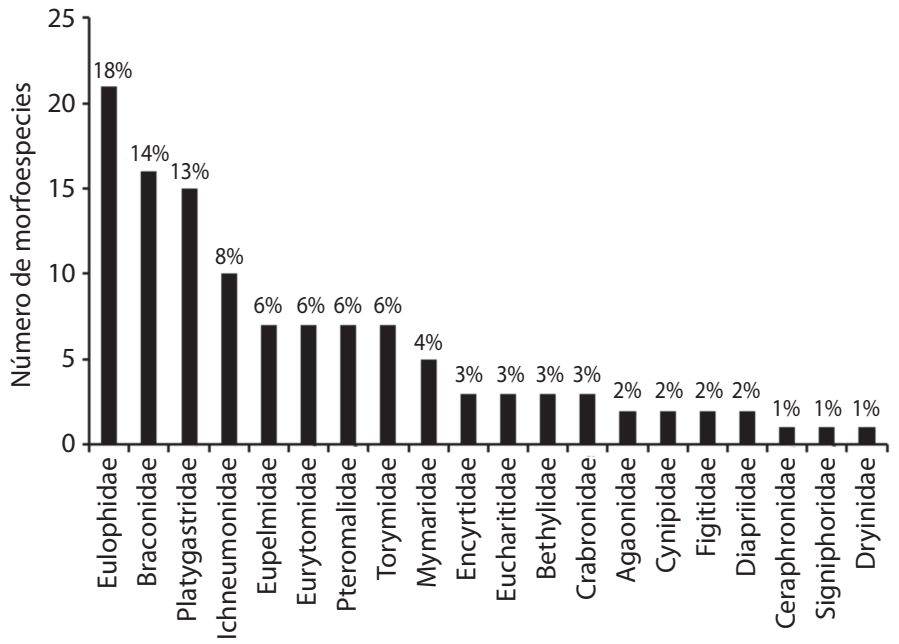

Fig. 1. Número de morfoespecies identificadas por familia.

Fig. 1. Number of morphospecies identified by family. 
CUADRO 1

Listado de morfoespecies de parasitoides registradas en la isla Gorgona

TABLE 1

List of parasitoid species recorded on Gorgona island

\begin{tabular}{|c|c|c|c|}
\hline Superfamilia/Familia & Subfamilia & Género/no. morfoespecies & Individuos \\
\hline \multicolumn{4}{|l|}{ Ceraphronoidea } \\
\hline Ceraphronidae & & Ceraphron sp. 1 & 7 \\
\hline \multicolumn{4}{|l|}{ Chalcidoidea } \\
\hline Agaonidae & Agaoninae & Pegoscapus sp. 1 & 2 \\
\hline Agaonidae & Agaoninae & Tetrapus sp. 1 & 1 \\
\hline Encyrtidae & Encyrtinae & Mucrencyrtus sp. 1 & 15 \\
\hline Encyrtidae & Encyrtinae & Mucrencyrtus sp. 2 & 2 \\
\hline Encyrtidae & Encyrtinae & Mucrencyrtus sp. 3 & 1 \\
\hline Eucharitidae & Eucharitinae & Kapala sp. 1 & 13 \\
\hline Eucharitidae & Eucharitinae & Obeza sp. 1 & 2 \\
\hline Eucharitidae & Oraseminae & Orasema sp. 1 & 1 \\
\hline Eulophidae & & 20 morfoespecies & 71 \\
\hline Eulophidae & Eulophinae & Eulophinae sp. 1 & 1 \\
\hline Eupelmidae & & 7 morfoespecies & 48 \\
\hline Eurytomidae & Eurytominae & Eurytoma sp. 1 & 14 \\
\hline Eurytomidae & Eurytominae & Eurytoma sp. 2 & 4 \\
\hline Eurytomidae & Eurytominae & Phylloxeroxenus sp. 1 & 5 \\
\hline Eurytomidae & Eurytominae & Sycophyla sp. 1 & 3 \\
\hline Eurytomidae & Eurytominae & Sycophyla sp. 2 & 17 \\
\hline Eurytomidae & Eurytominae & Tetramesa sp. 1 & 4 \\
\hline Eurytomidae & Rileynae & Rileya sp. 1 & 1 \\
\hline Mymaridae & Gonatocerinae & Gonatocerus sp. 1 & 3 \\
\hline Mymaridae & Gonatocerinae & Gonatocerus sp. 2 & 5 \\
\hline Mymaridae & Gonatocerinae & Gonatocerus sp. 3 & 7 \\
\hline Mymaridae & & Camptoptera sp. 1 & 1 \\
\hline Mymaridae & & Acmopolynema sp.1 & 1 \\
\hline Pteromalidae & & 6 morfoespecies & 27 \\
\hline Pteromalidae & Colotrechninae & Dipachystigma sp. 1 & 2 \\
\hline Signiphoridae & & Signiphoridae sp. 1 & 3 \\
\hline Torymidae & Toryminae & Torymus sp. 1 & 10 \\
\hline Torymidae & Toryminae & Idiomacromerus sp. 1 & 8 \\
\hline Torymidae & Toryminae & Pseudotorymus sp. 1 & 3 \\
\hline Torymidae & Toryminae & Microdontomerus sp. 1 & 1 \\
\hline Torymidae & Toryminae & Torymus sp. 2 & 1 \\
\hline Torymidae & Toryminae & Microdontomerus sp. 2 & 1 \\
\hline Torymidae & Toryminae & Glyphomerus sp. 1 & 3 \\
\hline \multicolumn{4}{|l|}{ Chrysidoidea } \\
\hline Bethylidae & Pristocerinae & Pristocerinae sp. 1 & 19 \\
\hline Bethylidae & Pristocerinae & Pristocerinae sp. 2 & 4 \\
\hline Bethylidae & Pristocerinae & Pristocerinae sp. 3 & 1 \\
\hline Crabronidae & Miscophini & Crabronidae sp. 1 & 1 \\
\hline Crabronidae & Miscophini & Crabronidae sp. 2 & 1 \\
\hline Crabronidae & Psenini & Crabronidae sp. 3 & 1 \\
\hline
\end{tabular}


CUADRO 1 (Continuación) / TABLE 1 (Continued)

\begin{tabular}{|c|c|c|c|}
\hline Superfamilia/Familia & Subfamilia & Género/no. morfoespecies & Individuos \\
\hline Dryinidae & Anteoninae & Metanteon sp. 1 & 1 \\
\hline \multicolumn{4}{|l|}{ Cynipoidea } \\
\hline Cynipidae & & Eschatocerus sp. 1 & 8 \\
\hline Cynipidae & & Cynipidae sp. 1 & 3 \\
\hline Figitidae & Eucoilinae & Ganaspidium sp. 1 & 11 \\
\hline Figitidae & Eucoilinae & Ganaspidium sp. 2 & 5 \\
\hline \multicolumn{4}{|l|}{ Diaprioidea* } \\
\hline Diapriidae & Diapriinae & Diapriinae sp. 1 & 1 \\
\hline Diapriidae & Diapriinae & Diapriinae sp. 2 & 2 \\
\hline \multicolumn{4}{|l|}{ Ichneumonoidea } \\
\hline Braconidae & & 16 morfoespecies & 137 \\
\hline Ichneumonidae & & Ichneumonidae sp. 1 & 3 \\
\hline Ichneumonidae & & Ichneumonidae sp. 2 & 1 \\
\hline Ichneumonidae & & Ichneumonidae sp. 3 & 1 \\
\hline Ichneumonidae & Campopleginae & Campopleginae sp. 1 & 1 \\
\hline Ichneumonidae & Cryptinae & Cryptinae sp. 1 & 3 \\
\hline Ichneumonidae & Ichneumoninae & Ichneumoninae sp. 1 & 1 \\
\hline Ichneumonidae & Metopiinae & Metopiinae sp. 1 & 1 \\
\hline Ichneumonidae & Orthocentrinae & Orthocentrinae sp. 1 & 9 \\
\hline Ichneumonidae & Orthocentrinae & Orthocentrinae sp. 2 & 1 \\
\hline Ichneumonidae & Pedunculinae & Pedunculinae sp. 1 & 2 \\
\hline \multicolumn{4}{|l|}{ Platygastroidea* } \\
\hline Platygastridae & Platygastrinae & 5 morfoespecies & 52 \\
\hline Platygastridae & Scelioninae & 10 morfoespecies & 83 \\
\hline
\end{tabular}

*sensu Sharkey, 2007.

Las morfoespecies de Diaprioidea, Ichneumonoidea y Platygastroidea se determinaron solo hasta subfamilia. Diapriidae presentó dos especies de Diapriinae. En Ichneumonidae, se identificó una especie de Cryptinae, dos de Orthocentrinae y una especie para cada una de las subfamilias Metopiinae, Ichneumoninae, Campopleginae y Pedunculinae. En Platygastridae se identificaron cinco morfoespecies de la subfamilia Platygastrinae y diez de Scelioninae (Cuadro 1).

Los estimadores no paramétricos indicaron que la riqueza estimada oscila entre 163 y 170 especies de himenópteros parasitoides y se calculó una eficiencia promedio de muestreo de $71 \%$ (Cuadro 2). Del mismo modo, se estimó que el 41\% (48/118) de las especies identificadas tendrían una única aparición y el 19\% (22/118) solo dos ejemplares (Cuadro 2). En el Cuadro 3 se compara la riqueza de himenópteros parasitoides recolectados en el PNN Gorgona, con los valores conocidos para Colombia y el neotrópico. Las 118 especies y morfoespecies encontradas representan el 51.3\% (118/230) de

\section{CUADRO 2}

Valores de los estimadores no paramétricos de riqueza y eficiencia de muestreo

TABLE 2

Values of the non-parametric estimators of richness and sampling efficiency

\begin{tabular}{lcc} 
Estimador & $\begin{array}{c}\text { Número de } \\
\text { especies }\end{array}$ & $\begin{array}{c}\text { Eficiencia de } \\
\text { muestreo (\%) }\end{array}$ \\
ICE & 170.19 & 69.33 \\
Chao 2 & 163.98 & 80.61 \\
Jacknife 1 & 163 & 72.39 \\
Promedio & 165.72 & 71.20 \\
Especies únicas & 48 & \\
Especies duplicadas & 22 & \\
\hline
\end{tabular}


las especies registradas para el país y el 1.2\% (118/9081) de las neotropicales.

\section{DISCUSIÓN}

De las 20 familias recolectadas, sólo cinco de las familias habían sido reportadas para el PNN Gorgona: Eucharitidae, Mymaridae, Pteromalidae, Dryinidae y Braconidae (Fernández, 1995; Olmi, Virla \& Fernández, 2000; Sharkey, 2007; Noyes, 2012), indicando que 15 de las familias determinadas en el presente estudio son nuevos reportes para esta área protegida. Del mismo modo, 12 de los géneros identificados son nuevos registros para la isla; por lo tanto, se amplía el rango de distribución en Colombia de: Mucrencyrtus (Encyrtidae), Obeza (Eucharitidae), Phylloxeroxenus, Sycophyla y Tetramesa (Eurytomidae), Acmopolynema y Camptoptera (Mymaridae), Glyophomerus, Idiomacromerus, Microdontomerus y Pseudotorymus (Torymidae) y Metanteon (Dryinidae).

El primer listado de especies de Hymenoptera para Colombia, publicado por Fernández (1995), reporta 306 especies y se estiman otras 138. Actualmente, la base de datos mundial de Chalcidoidea, lista más de 5400 especies de esta superfamilia para el neotrópico, de las cuales 346 especies se encuentran en Colombia (Noyes, 2012; Cuadro 3), lo cual comprueba la falta de estudio de esta superfamilia en los últimos 15 años. En Chalcidoidea se encuentran los grupos más difíciles de identificar, no solo por su pequeño tamaño sino también por la falta de claves de identificación para las especies tropicales (Krombein, Hord, Smith \& Burks, 1979; Gibson et al., 1997; Sharkey, 2007). Consecuentemente, es de gran importancia incentivar la investigación de dichos grupos, ya que conocerlos es el primer paso para entender las complejas relaciones que los rodean (González et al., 2012). El registro de diez familias y 20 géneros de Chalcidoidea para el PNN Gorgona, permite acercarse aun más a la diversidad estimada de parasitoides calcidóideos de la isla y ampliar el registro de la superfamilia en el país.
CUADRO 3

Comparación entre el número de especies de parasitoides registradas en la isla Gorgona y las reportadas para Colombia y el neotrópico

TABLE 3

Comparison between the number of parasitoid species recorded on Gorgona island and the reported species for Colombia and the neotropics

\begin{tabular}{|c|c|c|c|}
\hline Superfamilia/Familia & Gorgona* & Colombia & Neotrópico \\
\hline \multicolumn{4}{|l|}{ Ceraphronoidea } \\
\hline Ceraphronidae & 1 & 2 & $>50$ \\
\hline \multicolumn{4}{|l|}{ Chalcidoidea } \\
\hline Agaonidae & 2 & 6 & 133 \\
\hline Encyrtidae & 3 & 70 & $>500$ \\
\hline Eucharitidae & 3 & 9 & 40 \\
\hline Eulophidae & 21 & 103 & 227 \\
\hline Eupelmidae & 7 & 8 & 85 \\
\hline Eurytomidae & 7 & 29 & 125 \\
\hline Mymaridae & 5 & 13 & 177 \\
\hline Pteromalidae & 7 & 43 & 242 \\
\hline Signiphoridae & 1 & 5 & 28 \\
\hline Torymidae & 7 & 11 & 60 \\
\hline \multicolumn{4}{|l|}{ Chrysidoidea } \\
\hline Bethylidae & 3 & 12 & 437 \\
\hline Crabronidae & 3 & & 1537 \\
\hline Dryinidae & 2 & 128 & 1046 \\
\hline \multicolumn{4}{|l|}{ Cynipoidea } \\
\hline Cynipidae & 2 & & 15 \\
\hline Figitidae & 2 & & 225 \\
\hline \multicolumn{4}{|l|}{ Diaprioidea } \\
\hline Diapriidae & 1 & 4 & 229 \\
\hline \multicolumn{4}{|l|}{ Ichneumonoidea } \\
\hline Braconidae & 16 & 46 & 1500 \\
\hline Ichneumonidae & 10 & 98 & 3137 \\
\hline \multicolumn{4}{|l|}{ Platygastroidea } \\
\hline Platygastridae & 15 & 15 & 454 \\
\hline
\end{tabular}

*Cantidad de morfoespecies identificadas en este estudio.

*Number of morphospecies identified in this study.

Braconidae fue la familia dominante en el área muestreada y la segunda más diversa con 16 morfoespecies, después de Eulophidae (21 morfoespecies). Lo anterior es consecuente con el número de especies de Braconidae reportadas para la región tropical y que la ubican como la segunda familia de parasitoides más diversa después de Ichneumonidae (Krombein et al., 1979; Noyes, 2012). Por su parte, 
de Eulophidae la familia con mayor número de morfoespecies identificados pero con una abundancia mucho menor, se podría esperar que el PNN Gorgona conservara aproximadamente un $10 \%$ de las especies del neotrópico (Fernández \& Sharkey, 2006; Noyes, 2012).

Se han registrado seis subfamilias, 22 géneros y 1046 especies de Dryinidae en la región neotropical y en particular el género Metanteon, no había sido reportado para Colombia (Olmi et al. 2000); solo se conocía de Argentina la especie M. aerias (Walker, 1839). Los drí́nidos neotropicales por ser poco estudiados, son de gran valor taxonómico; sin embargo, en países como Colombia, Venezuela y Perú, la falta de muestreos no ha permitido registrar muchas de sus especies (Fernández, 1995; Olmi et al., 2000). El estudio de la familia Diapriidae ha sido limitado en Colombia y la mayoría de las especies reportadas provienen de la región andina y son escasas las registradas para el pacífico colombiano (Fernández, 1995; Masner \& García, 2000). En el presente estudio, el muestreo se llevó a cabo por un número limitado de días (tres); por lo cual expandir el esfuerzo de muestreo en la zona podría traer consigo el registro de un número aún mayor de himenópteros parasitoides y de aquellos pertenecientes a Vespoidea. La ampliación de distribución de esta familia hasta la zona pacífica abre las puertas para posteriores investigaciones. Se recomienda la realización nuevos muestreos enfocados en diferentes épocas del año, diferentes lugares de la isla y el uso de diferentes técnicas de muestreo (colección de hojarasca y trampas de caída).

Los himenópteros parasitoides colectados en el PNN Gorgona (118 morfoespecies) representan aproximadamente el $51 \%$ de las especies registradas hasta la fecha para Colombia. Estos valores de riqueza pueden atribuirse a la heterogeneidad de hábitats, resultado de una gran diversidad de especies de plantas y los insectos que éstas resguardan, los cuales pueden servir de hospederos para este amplio gremio (Basset, Aberlenc \& Delvare, 1992; González et al., 2012). Adicionalmente, los himenópteros parasitoides presentan diferentes asociaciones con otras especies no fitófagas como insectos coprófagos, carroñeros, saprófagos y muchos otros, actuando como importantes organismos reguladores de poblaciones de otros insectos en el ecosistema (LaSalle, 1993). Por lo tanto, se confirma que el PNN Gorgona es una zona de gran diversidad de insectos; y que Hymenoptera por ser un orden megadiverso, es uno de los más representativos de su ecosistema. Se recomienda continuar con la determinación específica de los himenópteros parasitoides de la isla Gorgona y muestrear otros de sus hábitats.

\section{AGRADECIMIENTOS}

A. Andrew C. Mason y F. Montealegre-Z. (Universidad de Toronto) por permitirnos trabajar y conservar muestras de artrópodos colectados en el PNN Gorgona, durante el desarrollo de su proyecto de investigación financiado por la National Geographic ( ${ }^{\circ}$. 7928-05) y la National Science and Engineering Research Council Canadá (Nº. 238882). Fabio A. Sarria y María Cleopatra Pimienta participaron en el trabajo de campo. La Unidad Administrativa de Parques Nacionales Naturales de Colombia concedió el permiso de colecta DTSO-G-31. Julian Mendivil colaboró en la determinación de algunos individuos. Tres evaluadores anónimos aportaron correcciones al manuscrito.

\section{RESUMEN}

A pesar de la reconocida riqueza de Colombia, los estudios de la fauna de himenópteros son muy incipientes, fragmentarios y no hay revisiones de base que muestren el tamaño y composición de la fauna del país. Los himenópteros parasitoides son muy comunes y abundantes en todos los ecosistemas terrestres, actuando como reguladores de poblaciones de otros insectos. Este trabajo fue dirigido hacia la identificación de especies asociadas al dosel del bosque húmedo tropical del Parque Nacional Natural Gorgona, ecosistema insular ubicado en la parte sur del Pacífico colombiano, con el propósito de ampliar el conocimiento sobre este orden. En noviembre de 2007, se muestrearon 16 árboles mediante la técnica de nebulización, utilizando un insecticida piretroide biodegradable aplicado desde $1 \mathrm{~m}$ sobre el nivel del suelo hasta el dosel. Se examinaron 641 individuos (identificados $45 \%$ a subfamilias y $28 \%$ a género) pertenecientes a siete superfamilias, 
20 familias y 118 morfoespecies, siendo Chalcidoidea la superfamilia más representativa, Eulophidae la familia más diversa y Braconidae la familia dominante con el mayor número de ejemplares colectados. Se reportan 12 géneros nuevos para la isla, ampliando su rango de distribución y posicionando este estudio como el primero enfocado en este gremio de Hymenoptera en el parque.

Palabras clave: Chalcidoidea, avispas, ecosistema insular, técnica de termonebulización, bosque húmedo tropical.

\section{REFERENCIAS}

Aguirre, J. C. \& Rangel, O. C. (1990). (Eds.). Biota y ecosistemas de Gorgona. Editorial Fondo FEN, Bogotá, Colombia.

Andrade, G., Cadena, A., Gómez-Laverde, M., Uribe, J. \& Benavides, P. (1990). Artrópofauna asociada al suelo. In J, Aguirre \& O. Rangel (Eds.), Biota y ecosistemas de Gorgona (pp. 91-106). Fondo FEN, Bogotá, Colombia.

Arias-Penna, D. C. (2007). New geographical records of the genus Urosigalphus Ashmead, 1889 (Hymenoptera: Braconidae, Heliconinae) for Colombia. Boletín del Museo de Entomología, 8: 1-9.

Baena, M. L. (1993). Hormigas cazadoras del género Pachycondyla (Hymenoptera: Formicidae) de la isla Gorgona y la planicie pacífica colombiana. Boletín del Museo de Entomología, 1: 13-21.

Basset, Y., Aberlenc, H. P. \& Delvare, G. (1992) Abundance and stratification of foliage arthropods in a lowland rain forest of Cameroon. Ecological Entomology, 17:310-318.

Basset, Y., Hammond, P. M., Barrios, H., Holloway, J. D. \& Miller, S. E. (2003). Vertical stratification of arthropod assemblages. In Y. Basset, V. Novotny, S. E. Miller \& R. L. Kitching (Eds.), Arthropods of Tropical Forests (pp. 17-27). Cambridge University, United Kingdom.

Cavelier, J. \& Yockteng, R. (1998). Diversidad y mecanismos de dispersión de árboles de la isla Gorgona y de los bosques húmedos tropicales del pacífico Colombo-Ecuatoriano. Revista de Biología Tropical, 46: 45-53.

Chamorro, C. (1990). Suelos. In J. Aguirre \& J.O. Rangel (Eds.), Biota y ecosistemas de Gorgona (pp.65-72). Fondo FEN, Bogotá, Colombia.

Colwell, R. (2012). Estimates statistical estimation of species richness and shared species from samples, Version 8.2. Retrieved from www.viceroy.eed.uconn. edu/estimates

Erwin, T. L. \& Scott, J. C. (1980). Seasonal size patterns, trophic structure, and richness of Coleoptera in the arboreal ecosystem: the fauna of the tree Luehea seemanii Triana and Planch in the Canal Zone of Panama. The Coleopterists Bulletin, 34: 305-322.

Erwin, T. L. (1982). Tropical forests: Their richness in Coleoptera and other Arthropod species. The Coleopterists Bulletin, 36: 74-75.

Espinal, L .S. \& Montenegro, E. (1977). Zonas de vida o formaciones vegetales de Colombia. Memoria explicativa sobre el mapa ecológico. IGAC, 13 (11). Bogotá, Colombia.

Fernández, F. (1995). La diversidad de los Hymenoptera en Colombia. In J. Orlando \& C. Rangel (Ed.), Colombia diversidad biótica I (pp. 373-442). Guadalupe LTDA, Bogotá, Colombia.

Fernández, F. (2001). Checklist of genera and subgenera of Aculeate Hymenoptera of the neotropical region (Hymenoptera: Vespomorpha). Biota Colombiana, 2(2): 87-130.

Fernández, F. \& Sharkey, M. J. (2006). (Eds.). Introducción a los Hymenoptera de la región neotropical. Sociedad Colombiana de Entomología y Universidad Nacional de Colombia. Guadalupe LTDA, Bogotá, Colombia.

Gaston, K. (1991). The magnitude of global insect species richness. Conservation Biology, 5:183-186.

Gibson, G. A. P., Huber, J. T. \& Woolley, J. B. (1997). (Eds.). Annotated keys to the genera of Nearctic Chalcidoidea (Hymenoptera). Ottawa, Ontario, Canadá.

Giraldo, A. (2012). Geomorfología e hidroclimatología de isla Gorgona. In A. Giraldo \& B. Valencia (Eds.), Isla Gorgona paraíso de biodiversidad y ciencia (pp. 17-23). Programa Editorial Universidad del Valle, Cali, Colombia.

González-Córdoba, M., González, V. H. \& MontoyaLerma, J. (2011). Composición taxonómica, distribución y patrón de forrajeo de abejas (Hymenoptera: Apoidea) en el PNN Gorgona, pacífico colombiano. In J. Sewlal (Ed.), Living World, Journal of the Trinidad \& Tobago field naturalist's club. Universidad de las Indias Occidentales, San Agustín, Trinidad \& Tobago.

González, R., Armbrecht, I., Montoya-Lerma, J., Carrejo, N., Zuñiga, M. C., Chacón, P., Posso, C. E., Torres, D., Calero, H., Mendivil, J., González, M., Valdés, S., Sarria, F. \& Cardona, W. (2012). In A. Giraldo \& B. Valencia (Eds.), Isla Gorgona, Paraíso de biodiversidad y ciencia (pp. 149-191). Programa editorial de la Universidad del Valle, Cali, Colombia.

Kitching, R. L., Mitchell, H., Morse, G. \& Thebaud, C. (1997). Determinants of species richness in assemblages of canopy arthropods in rainforests. In N. E. Stork, J. A. Adis \& R. K. Didham (Eds.), Canopy Arthropods (pp. 131- 150). Chapman and Hall, London, England. 
Krombein, K. V., Hord, P. D., Smith, D. R. \& Burks, B. D. (1979). Catalog of Hymenoptera in America North of Mexico. Vol. 1. Smithsonian Institution, Washington, D.C.

LaSalle, J. (1993). Parasitic Hymenoptera, Biological Control and Biodiversity. In J. LaSalle \& I. D. Gauld (Eds.), Hymenoptera and biodiversity (pp. 197-215). CABI. United Kingdom.

LaSalle, J. \& Gauld, I. D. (1993). Hymenoptera: their diversity, and their impact on the diversity of other organisms. In J. LaSalle \& I. D. Gauld (Eds.), Hymenoptera and biodiversity (pp. 1-26). CABI. United Kingdom.

López, L., Armbrecht, I., Montoya-Lerma J. \& Molina, E. J. (2013). Diversidad de avispas parasitoides en un sistema silvopastoril orgánico de producción ganadera de Colombia. REVAIA, 17(1): 65-78. Retrieved from www.ucol.mx/revaia/portal/pdf/2013/enero/3. pdf

Lucky, A., Erwin, T. L. \& Witman, J. D. (2002). Temporal and spatial diversity and distribution of arboreal Carabidae (Coleoptera) in a Western Amazonian rain forest. Biotrópica, 34: 376-386.

Madrigal-Cardeño, A. (2001). Fundamentos de Control Biológico de Plagas. Universidad Nacional de Colombia, Medellín, Colombia.

Masner, L. \& García, J. L. (2000). The genera of Diapriinae (Hymenoptera: Diapriidae) in the new world. Bulletin of the American Museum of Natural History, 268: 1-138.

Noyes, J. S. (2012). (última actualización: junio 2012). Universal Chalcidoidea Database. The Natural History Museum, New York, USA. Retrieved from www. nhm.ac.uk/jdsml/research-curation/research/projects/ chalcidoids/

Olmi, M., Virla, E. G. \& Fernández, F. (2000). Las avispas Dryinidae de la región neotropical (Hymenoptera: Chrysidoidea). Biota Colombiana, 1(2):141-163.

Pimienta, M. C. (2005). Protocolo para la caracterización de insectos de dosel. (Informe No. 2, contrato de prestación de servicios No. 173/04). Instituto Alexander von Humboldt, Bogotá, Colombia.

Pimienta, C., Montealegre, F., Chacón de Ulloa, P., González, R. \& Mejía, J. (2008). Diversidad de artrópodos arborícolas en el PNN Gorgona (Cauca, Colombia). Resúmenes XXXV Congreso de la Sociedad Colombiana de Entomología, Cali, Colombia.
Ramírez, S., Dressler, R. L. \& Ospina, M. (2002). Abejas Euglosinas (Hymenoptera: Apidae) de la región neotropical: listado de especies con notas sobre su biología. Biota Colombiana, (3): 7-118.

Rangel, J. O. (1990). Tipos de vegetación. In J. Aguirre \& O. Rangel (Eds.), Biota y ecosistemas de Gorgona (pp. 106-126). Fondo FEN, Colombia.

Sharkey, M. J. (2007). Phylogeny and classification of Hymenoptera. Zootaxa, 1668: 521-548. Retrieved from www.mapress.com/zootaxa/2007f/ zt01668p548.pdf

Stork, N. E. (1993). How many species are there?. Biodiversity and Conservation, 2: 215-232.

Stork, N. E. \& Hammond, P. M. (1997). Sampling arthropods from tree-crowns by fogging with knockdown insecticides: lessons from studies of oak tree beetle assemblages in Richmond Park (UK). In N. E. Stork, J. A. Adis \& R. K. Didham (Eds.), Canopy Arthropo$d s$ (pp. 3-26.). Chapman and Hall, London, England.

Ugarte-Lavados, Y. \& Dassori, P. (2009). Changes in abundances of parasitoids (Hymenoptera) and others arthropods (Araneae) in managed compared unmanaged native rainforests. In N. Verhoest, P. Boeckx, C. Oyarzún \& R. Godoy (Eds.), Ecological advances on Chilean temperate rainforests, Chapter: VI (pp. 65-88). Academia Press, Universidad Austral de Chile, Chile.

Ulloa-Chacón, P. (1990). Biologie de la reproduction chez la petite fourmi de feu Wasmannia auropunctata (Roger) (Hymenoptera: Formicidae) (Doctoral dissertation). Université de Lausanne, Switzerland.

Valdés-Rodríguez, S., Hurtado, A. \& Chacón, P. (2010). Diversidad de hormigas (Hymenoptera: Formicidae) arbóreas en el PNN Gorgona (Cauca, Colombia). Ponencia presentada en XXXVII Congreso de la Sociedad Colombiana de Entomología, Bogotá, Colombia.

Vásquez Vélez, A. I., Cortés-Ceballos, L., Calero-Rosales, V. E., Soto, E. \& Torres, A. M. (2012). La vegetación en el Parque Nacional Natural Gorgona. In A. Giraldo \& B. Valencia (Eds.), Isla Gorgona paraíso de la biodiversidad y ciencia (pp. 133-147). Programa editorial Universidad del Valle, Cali, Colombia.

Wilson, E. O. (1988). The current state of biological diversity. In E. O.Wilson (Ed.), Biodiversity (p. 3-17). National Academy, Washington D.C., USA. 
